\title{
Las perspectivas transnacionales para el análisis de la protección social en contextos migratorios
}

\author{
Transnational perspectives for analyzing social protection in the context of \\ migration
}

\author{
Sònia Parella \\ Universitat Autònoma de Barcelona, España \\ sonia.parella@uab.cat
}

Thales Speroni

Universitat Autònoma de Barcelona, España

Universidade Federal do Rio Grande do Sul, Brasil

thales.speroni@ufrgs.br

\begin{abstract}
Resumen
Vivimos en una época marcada por un profundo desencaje entre una vida social cada vez más transnacional, y unos recursos de acceso a la protección social que siguen, en gran medida, vinculados al estado nacional moderno. En respuesta a eso, las familias transnacionales usan la movilidad internacional como medio para ampliar sus posibilidades de acceso a recursos de protección social prestados por los estados de bienestar o generados a través de la circulación de cuidado y el envío de remesas. En este artículo se presenta la agenda de investigación propuesta por la Protección Social Transnacional, enfatizando su vínculo con las investigaciones sobre el cuidado y la familia transnacional, así como el diálogo con los estudios de los regímenes de bienestar, desarrollo y políticas sociales globales.
\end{abstract}

Palabras claves: Protección Social Transnacional, Familias Transnacionales, Organización Social de los Cuidados, Regímenes de Bienestar

\begin{abstract}
We live in an age marked by a central paradox: social life is increasingly transnational, while the primary access to resources regarding social protection continue, to a large extent, to be linked to the modern nation-state. In response to this, transnational families use international mobility to expand the possibilities of accessing social protection through resources provided by welfare states, or that which is generated through the circulation of care and remittances transfers. In this article, we present the Transnational Social Protection research agenda, emphasizing its ties to research on transnational care and family, as well as how it dialogues with studies on welfare regimes, development and global social policy.
\end{abstract}

Key Words: Transnational Social Protection, Transnational Families, Social Organization of Care, Welfare Regimes 


\section{Introducción}

Vivimosenunaépocamarcadaporunprofundodesencaje entre una vida social cada vez más transnacional, y unos recursos de acceso a la protección social que siguen, en gran medida, vinculados al estado nacional moderno. El keynesianismo-westphaliano ha dejado de ser un marco satisfactorio para la comprensión del mundo presente, lo que conlleva importantes implicaciones sobre cómo pensamos la justicia (Fraser, 2009), el estado de bienestar (Bommes y Geddes, 2000; Levitt y de la Dehesa, 2003; 2017), las políticas públicas (Deacon et al, 1997; Yeates, 2014), la protección social (Faist, 2013; Boccagni, 2016; Levitt et al, 2016a) y las migraciones (Basch et al, 1994; Faist, 2000; Parella y Cavalcanti, 2008). Sin embargo, esta inconsistencia no es estática y los ejemplos de reacciones “desde abajo" y "desde arriba" son numerosos y diversificados, si bien todavía contingentes y dispersos. Por una parte, los individuos y sus familias buscan, a través de dinámicas multisituadas de movilidad e inmovilidad, el desarrollo de estrategias de protección social. Por otro lado, los estados nacionales, las organizaciones internacionales, las entidades transestatales y las organizaciones de la sociedad civil, ponen a disposición algunos recursos (todavía limitados) de protección social a escala transnacional.

Este complejo y dinámico rompecabezas es una de las razones que explica la creciente presencia de la protección social en los discursos públicos, así como el significativo interés académico por el tema (Bilecen y Barglowski, 2015a: 203). No obstante, todavía son insuficientes los diálogos entre las perspectivas que tratan el bienestar a escala nacional, los análisis de las políticas sociales globales y los abordajes del cuidado transnacional. La agenda de investigación sobre la Protección Social Transnacional (PST) emerge de la necesidad de construir esas sinergias por medio de un marco heurístico multidimensional, que sea capaz de combinar el análisis de regímenes, redes y estrategias de cuidado con el de las configuraciones de bienestar y de desarrollo de políticas sociales globales. De ese modo, la PST busca analizar la provisión y el uso de la protección social a partir de múltiples escalas sociales (global, transnacional, internacional, nacional, subnacional y local) y desde diferentes esferas (los estados nacionales, los mercados, las organizaciones de la sociedad civil y las familias).

La PST puede ser entendida como el ensamblaje multisituado de elementos formales e informales que permiten el acceso a recursos tangibles e intangibles para hacer frente a los riesgos sociales en las esferas del cuidado, trabajo, salud y educación (Barglowski et al, 2015: 216; Bilecen y Barglowski, 2015a). A partir del esfuerzo de investigadores como Peggy Levitt y Thomas Faist, la PST propicia la relectura de los estudios anteriores (en particular los referentes a las familias transnacionales), así como el desarrollo de nuevas investigaciones (por ejemplo, Bilecen y Barglowski, 2015b; Boccagni, 2011; Levitt et al, 2016b; Herrera y Grijalva, 2017; Speroni, 2017; VivasRomero, 2017).

En este artículo se presenta, a modo de estado de la cuestión, la agenda de investigación propuesta por la PST. Nuestro objetivo es enfatizar su capacidad de movilizar simultáneamente una mirada ascendente (bottom-up) y descendente (top-down) sobre la protección social. De este modo, en la primera parte, ofrecemos una síntesis sobre los principales aportes de la literatura sobre las familias transnacionales; en la segunda parte, discutimos acerca de los estudios de los regímenes de bienestar y de las políticas sociales globales. Al final de la segunda parte se presentan las principales categorías y marcos interpretativos de la PST que permiten construir interfaces entre los campos investigativos previamente presentados. 


\section{Familia transnacional y protección social: potencial y limitaciones}

La literatura que subraya la familia como núcleo central de la construcción, mantenimiento y gestión de los proyectos migratorios, pone énfasis en la movilidad internacional como estrategia de protección social (Avato et al, 2010; Baldassar y Merla, 2014; Bryceson y Vuorela, 2002; Faist et al, 2015; SabatesWheeler y Feldman, 2011). Así, cuando se habla de familia transnacional y de los cuidados en relación con la movilidad más allá de las fronteras, de acuerdo con Gonzálvez (2016), un gran número de investigaciones se han centrado en la gestión del bienestar familiar con la mirada puesta en las zonas de origen.

Sin embargo, el interés en la familia transnacional como objeto de estudio, que se inicia en los años noventa, haido evolucionando en lo concerniente al marco conceptual desde el que aproximarse a sus configuraciones y efectos. Surge con fuerza de la mano del boom de investigaciones en torno al transnacionalismo (Basch et al, 1994). Con anterioridad, tal como subraya Kofman (2004), a pesar de la importancia de la familia a la hora de comprender las migraciones, se había generado escasa investigación en torno al vínculo entre familia y migraciones, en términos de estrategias desplegadas a lo largo del curso migratorio, relaciones de género, posiciones específicas de sus miembros y, especialmente, de sus implicaciones para las políticas públicas.

El concepto de familia transnacional más utilizado es el propuesto por Bryceson y Vuorela (2002: 2), que la define como aquella "estructura familiar cuyos miembros viven una parte o la mayor parte del tiempo separados los unos de los otros, siendo capaces de crear vínculos que permiten que sus miembros se sientan parte de una unidad y perciban su bienestar desde una dimensión colectiva, a pesar de la distancia física”. De dicha definición se desprende que el elemento clave de la estructura familiar transnacional es la existencia de un sentimiento de pertenencia por parte de sus miembros y que éstos participen colectivamente en la gestión del bienestar común (Levitt y Glick Schiller, 2004). Tales vínculos y lealtades pueden mantenerse, reforzarse o bien debilitarse, en la medida que los miembros migrantes decidan reagrupar, asentarse de forma permanente, regresar; o bien iniciar nuevas relaciones afectivas que puedan alterar las pertenencias a la estructura familiar transnacional que en su momento configuraron (Parella, 2012).

De acuerdo con Nina Sørensen (2007: 24), la vida de la familia transnacional "debe verse como algo influido por procesos económicos, políticos y sociales complejos e interconectados". Más allá de la agencia de los individuos, operan y constriñen factores estructurales que condicionan los patrones de movilidad de las personas (políticas migratorias, condiciones laborales en las zonas de destino, etc.) (Landolt, 2006). Los efectos de las normativas sobre la capacidad de movilidad de las familias tienen que ver, por ejemplo, con las restricciones jurídicas y económicas para llevar a cabo los procesos de reagrupación familiar. La migración por motivos familiares se enfrenta cada vez a mayores restricciones legales. Asimismo, el peso de las políticas migratorias también es sumamente relevante a la hora de explicar la capacidad de llevar a cabo las actividades de cuidado, principalmente en los casos de familias cuyos miembros carecen de documentos migratorios (dificulta las visitas a las zonas de origen y el cuidado de tipo presencial, lo que puede provocar mayor vulnerabilidad en aquellas familias que cuentan con miembros dependientes) (Gil Araujo, 2010; Hernández y Baca, 2016). De ese modo, los significados y prácticas de las familias transnacionales dependen directamente del estatus legal y los constreñimientos económicos, en la medida 
que son estos los que posibilitan/obstaculizan los intercambios transnacionales que pueden compensar la falta de intimidad y de presencia que entraña la distancia (Bonizzoni, 2012).

En la medida que la migración internacional se ha considerado un asunto masculino, la investigación sobre las configuraciones familiares derivadas de tales procesos de dispersión geográfica ha merecido escasa atención. Sin embargo, la expansión del uso de conceptos como "familia transnacional" o "maternidad transnacional" se sitúa a finales de los noventa, de la mano de investigaciones que, desde una perspectiva de género, analizan las dinámicas de globalización del cuidado que generan las estructuras familiares transnacionales (Pérez-Orozco 2010). La mayor parte de estos trabajos se enmarcan en la perspectiva de las cadenas globales de cuidados (Hochschild, 2000, 2005; Parreñas, 2005). Dicha perspectiva evidencia un sistema global de explotación que favorece la migración de las mujeres del Sur hacia el Norte para trabajar en el sector formal e informal de los cuidados, debiendo dejar a sus propios hijos al cuidado de otros miembros de su familia (generalmente mujeres) o de trabajadoras remuneradas. En el caso específico de muchos países de América Latina y el Caribe, de acuerdo con Molano (et al 2012), se consolida a partir de los noventa una modalidad de migración protagonizada por mujeres que se insertan en destino en el sector de cuidados, que emigran principalmente hacia países de la misma región, o hacia Europa o los Estados Unidos; lo hacen de manera autónoma, en calidad de pioneras de un proyecto migratorio de carácter familiar, aunque algunas emigran movidas por motivaciones propias, al margen de un proyecto familiar. Esta migración transforma y reorienta las relaciones conyugales y materno-filiales, con fuertes implicaciones en términos de desigualdades, así como de acceso a los recursos de protección social (Pedone, 2008; Parella y Oso, 2012).
Para el caso concreto de la migración latinoamericana en España, han proliferado diversas investigaciones sobre los hogares trasnacionales y sus estrategias en la provisión de remesas y cuidado, a menudo desde aproximaciones etnográficas multisituadas. Coincidiendo con los trabajos pioneros de HondagneuSotelo, (1994, 1999), focalizados en las migraciones de mujeres latinoamericanas hacia Estados Unidos, Laura Oso (1998) y Carmen Gregorio (1998) investigan la migración hacia España de las mujeres jefas de hogar, tomando en cuenta la organización de los grupos domésticos transnacionales en sus prácticas de producción y reproducción social, desde una perspectiva de género. Con posterioridad, cabe destacar, entre muchas otras investigaciones, los estudios de Pedone (2003, 2008), Herrera (2004, 2008) y Lagomarsino y Castellani (2016) para el caso ecuatoriano, así como el trabajo de Rivas, Gonzálvez et al (2009) sobre familias migrantes transnacionales que transitan entre España y Colombia. El contexto de la migración boliviana en España desde una mirada transnacional ha sido abordado por distintos trabajos que muestran los arreglos y formas de gestión de los vínculos transnacionales y sus impactos en las relaciones de género e intergeneracionales (Guaygua, et al, 2010; Tapia, 2011; Parella, 2012).

Las familias transnacionales usan la movilidad internacional como recurso para la protección social. Sin embargo, el trabajo de cuidado, puesto que se lleva a cabo principalmente en el ámbito familiar y es uno de los elementos constitutivos de dicha protección, se ve claramente alterado como consecuencia de la emigración femenina. De acuerdo con Hernández y Baca (2016), se configura un escenario complejo, condicionado por las políticas migratorias, las necesidades específicas de la unidad familiar y los roles de género. Los intercambios de cuidado están inmersos en construcciones de género que provocan desigualdad entre los miembros de la familia, en donde 
las mujeres aparecen como las principales proveedoras de cuidado en su papel de madres, esposas, hermanas o hijas (Herrera, 2011). Son diversos los estudios que subrayan la complejidad que supone para las mujeres migrantes la vivencia del proceso migratorio, tanto por los obstáculos y presiones psicológicas y legales, sino también por cómo tener que asumir los estereotipos que la visualizan como principal o única encargada del ámbito reproductivo. En consecuencia, su "abandono del hogar" se interpreta como conducta estigmatizada para con sus propias familias y sociedades de origen (Fuentes y Agrela 2015).

$\mathrm{Si}$ bien los estudios enmarcados dentro de la perspectiva de las cadenas globales de cuidados destacan principalmente los costes humanos y emocionales que conlleva el hecho de que las mujeres migrantes no puedan cuidar de sus hijos a distancia, esta visión unidimensional y física de los "cuidados" ha sido ampliamente cuestionada por la literatura más reciente (Baldassar et al, 2007; Baldassar y Merla, 2014; Baldassar y Merla, 2016; Merla 2014). Desde una concepción multidimensional de los cuidados como elemento esencial en el mantenimiento de las relaciones familiares a distancia, se incluye, de acuerdo con Merla (2014: 40), "no solo los cuidados personales, sino también el apoyo financiero (en forma de envío de dinero y de objetos), el apoyo práctico (intercambio de consejos, asistencia en las actividades de la vida cotidiana), el apoyo emocional y un largo etcétera". Estos tipos de apoyo no necesariamente pueden proporcionarse de forma presencial (mediante la copresencia física permanente o visitas esporádicas); sino también a través del uso de medios de comunicación como el teléfono, emails, las apps de internet, las redes sociales o Skype, que permiten la coordinación o gestión a distancia (Baldassar et al, 2007).

De ese modo, el cuidado transnacional implica un intercambio recíproco y asimétrico de flujos de cuidado que circulan en (y entre) las sociedades de origen y destino, en forma de bienes y servicios (Merla 2014). En dicho intercambio participan miembros de distintas generaciones y se involucran relaciones de cuidado de diferente naturaleza a lo largo del tiempo (Herrera, 2011; Merla, 2014). Esta visión es la principal aportación atribuible al marco conceptual en torno a la noción de «circulación de cuidados», elaborado por Baldassar y Merla (2014). Las autoras proponen completar el acercamiento político-económico de las cadenas globales de cuidados con otro centrado en torno a la "circulación transnacional". Plantean que los miembros de las redes familiares transnacionales pueden estar activa y pasivamente implicados en el intercambio recíproco y al tiempo asimétrico, de flujos de cuidados, en función de cuáles sean sus necesidades e intereses, así como de las estrategias a su alcance. Dichas estrategias dependerán de los contextos políticos, económicos, culturales y sociales en los que se inscriben, tanto en origen como en destino (Baldassar y Merla 2014; Merla 2014). Al respecto, Kilkey y Merla (2014) subrayan la necesidad de situar este enfoque de la circulación dentro de los marcos institucionales que imponen contingencias estructurales; en la medida que legitiman determinados modelos normativos de familia, ideologías de género o concepciones del cuidado a través de la política pública (en especial la política migratoria y las políticas sociales) y de los discursos institucionales (Gil Araujo y Pedone, 2014; Herrera, 2011, 2012; Pedone, Agrela y Gil Araujo, 2012; Pedone y Gil Araujo, 2008).

El enfoque de la circulación ofrece sofisticadas herramientas para analizar empíricamente la multidimensionalidad de los cuidados y la complejidad de las interacciones sociales que su gestión a distancia conlleva. Asimismo, proporciona instrumentos para captar las desigualdades de poder y las cuestiones de 
justicia social que tienen que ver con la circulación del cuidado. De ese modo, el amplio espectro de ajustes multidireccionales, de carácter formal o informal, que pueden tener lugar a lo largo de estos procesos, revelan los factores y matices que entran en juego a la hora de comprender las distintas posiciones de los actores que se imbrican dentro de las jerarquías de poder basadas en las intersecciones de género, raza, clase social, edad y ciclo vital que sostienen dicha circulación (Baldassar y Merla 2014; Parella, 2016). De ese modo, se concreta, en palabras de Baldassar y Merla (2014: 55), "la forma, intensidad y calidad de la participación de los actores implicados (o excluidos) de los circuitos de cuidado". En definitiva, la noción de circulación del cuidado puede ser considerada una forma de crear, mantener y transformar las estrategias de protección social a escala transnacional (Speroni, 2017).

El impacto de este enfoque ha sido importante y prolífico en el ámbito académico. Son numerosas las investigaciones que muestran concreciones empíricas específicas en cuanto a las asimetrías que puede comportar la "circulación de cuidados", analizadas desde todos los ángulos. A modo ilustrativo, el trabajo de Pérez y Neira (2017) analiza la realidad que viven las abuelas con menores a cargo en el cantón Sígsig (Ecuador), cuyas madres han iniciado un proyecto migratorio. Si bien estas abuelas practican el altruismo de cuidados y contribuyen a la protección de las niñas y niños "left-behind", se detectan importantes riesgos según perfiles, en cuanto a asimetrías y grados de exclusión. Si bien, en algunos casos, su rol les confiere autoridad en la toma de decisiones y en la gestión de los recursos (remesas) que llegan desde el exterior; en otros, cuando se enfrentan a la ausencia de ayudas tanto por parte de los hijos/as (no reciben remesas) como del estado, asumen lo que las autoras denominan "una "resignación solidaria" por tener que cuidar de forma "permanente" a los nietos (Pérez y Neira, 2017: 67), debiéndose enfrentar a menudo a graves riesgos de exclusión y a condiciones de vida sumamente precarias. En cualquiera de los casos, sienten que son ellas, en tanto que cuidadoras, las que posibilitan la supervivencia de un hogar multisituado (Pérez y Neira, 2017).

El foco en la familia transnacional y en la "circulación del cuidado" tiene importantes derivaciones para las políticas públicas en general $\mathrm{y}$, de modo específico, sobre la concepción del "social care" y las políticas sociales. El concepto de "social care" concibe el cuidado como una actividad o conjunto de relaciones constituidas a partir de la interacción entre el estado, el mercado, la familia y el tercer sector o comunidad (Daly y Lewis, 2000: 281). Este concepto se fundamenta en una noción del cuidado y de los derechos sociales fuertemente anclada en un estadonación territorializado. Es menester transcender los límites de los estados-nación, a base de incorporar los aspectos transnacionales del cuidado inherentes a la movilidad y a la separación geográfica, así como de incorporar las implicaciones de los ajustes de dicho cuidado transnacional como objeto de política social en sí mismos (Baldassar y Merla, 2014).

De acuerdo con Gonzálvez (2016), la “organización social de los cuidados" constituye una adaptación regional, surgida en América Latina, del concepto Social Care propuesto por Daly y Lewis, antes mencionado. En palabras de Arriagada (2010: 59), tal concepto se refiere a las “interrelaciones entre las políticas económicas y sociales del cuidado. Se trata de la forma de distribuir, entender y gestionar la necesidad de cuidados que sustentan el funcionamiento del sistema económico y de la política social'. De ese modo, tanto si se trata de provisión de cuidados transnacional como si no, se asume que ésta no transcurre de manera aislada o estancada, sino como resultante de una continuidad en la que se suceden actividades, trabajos y responsabilidades entre los distintos actores antes mencionados - a saber, familia, comunidad, mercado y estado - (Gonzálvez, 2016). 
Desde esta perspectiva, empiezan a surgir estudios que pretenden analizar cómo se organizan socialmente los cuidados a nivel transnacional, para los que la familia se convierte en una esfera de acción (situada al mismo nivel que las demás), que no actúa de manera aislada. Se trata de trabajos teórico-conceptuales y analíticos que van más allá de la distribución intrafamiliar de los cuidados y de las posiciones de género y parentesco dentro de las familias, para abarcar las distintas esferas de la protección social en la comprensión de las causas y el impacto de las migraciones (Gonzálvez, 2016). Este enfoque, centrado en la noción de protección social transnacional (PST) y la interrelación entre sus esferas, será abordado en el próximo apartado.

\section{La protección social transnacional}

\subsection{Hacia una nueva agenda de investigación}

La agenda de investigación de la PST emerge de la necesidad de convergencia entre, por un lado, las investigaciones sobre la familia y el cuidado transnacional y, por el otro los estudios sobre el bienestar social y las políticas sociales globales. Tal y como se desprende del apartado anterior, las investigaciones sobre las familias transnacionales tienden a enfocarse en los arreglos de cuidado y en la gestión de recursos internos a la familia, así como en los procesos de desigualdades de género y generacionales que emergen de tales dinámicas. Sin embargo, tal y como demuestra el concepto de "organización social de los cuidados" (Gonzálvez, 2016), esa tendencia ha ido cambiando; de modo que, cada vez más, nuevos estudios o relecturas de investigaciones anteriores abordan las dinámicas transnacionales del cuidado en términos de protección social.
En segundo lugar, el análisis de los regímenes de bienestar (Esping-Andersen, 1990) resulta parcial, por cuanto se circunscribe al estado nacional como unidad de análisis y considerar que los derechos derivan directamente de la ciudadanía. Por lo tanto, se dedica escasa atención a las implicaciones de las migraciones para la provisión y elegibilidad de la protección social (Sabates-Wheeler y Feldman, 2011: 15) y no se toma en consideración la posibilidad de que una persona combine recursos de protección procedentes de más de un estado nacional (Levitt et al, 2016a: 4). Además, el análisis en términos de regímenes de bienestar tiende a reducir la complejidad de las relaciones de cuidado y las interfaces entre los estados, el mercado, las comunidades y las familias (Bilecen y Barglowski, 2015a: 206). No obstante, es fundamental dialogar con las investigaciones sobre los regímenes de bienestar; en la medida que una parte importante de los estudios migratorios no incorpora los variados matices y contrastes entre distintos estados-nación (pero también subnacionales y locales) que definen el acceso de los migrantes a la protección social (Sabates-Wheeler y Feldman, 2011: 15; Dobbs y Levitt, 2017).

Por último, las investigaciones vinculadas a las políticas sociales globales exploran cómo los procesos de globalización impactan sobre las políticas sociales y los derechos. Se trata de estudios multidisciplinarios que tienen como foco la circulación y las interfaces de discursos y prácticas de los actores internacionales en lo que concierne a la política social y a sus efectos a escala nacional (Yeates, 2006: 17). Este enfoque se ha mostrado altamente provechoso para la comprensión de las múltiples escalas de las políticas públicas; en especial, para las vinculadas a la salud, educación, seguridad social, vivienda y trabajo. Sin embargo, deja fuera la acción de los actores no institucionalizados, como los individuos y las familias (Boccagni, 2016: 3; Levitt et al, 2016a: 4). 
La óptica transnacional, en general, así como la aplicada a la protección social en particular, abordan los distintos niveles de lo social de forma simultánea, movilizando tanto enfoques descendentes (top-down) que se pueden apreciar en las políticas sociales globales y los estudios de los regímenes de bienestar; como ascendentes (bottom-up), viéndose en las investigaciones sobre las familias transnacionales (Levitt y de la Dehesa, 2017: 1521). Esta intersección analítica permite comprender las interfaces entre regímenes de cuidado (con énfasis en los factores vinculados a género y generación), las dinámicas de movilidad (y no movilidad), los regímenes de bienestar y el desarrollo de políticas sociales. Así, la propuesta de la PST consiste en, por un lado, concebir el cuidado y las remesas transnacionales como componentes del bienestar social y, por otro lado, comprender los impactos de tales prácticas en las representaciones, prácticas y arreglos institucionales de bienestar (Boccagni, 2016: 3;7). La PST se sitúa precisamente en el punto de encuentro entre estas distintas agendas de investigación (Bilecen y Barglowski, 2015a).

Levitt, Viterna, Mueller y Lloyd (2016a) resumen las preguntas generales que guían la perspectiva de la PST: “¿Cuándo y cómo las personas en movimiento son protegidas y proporcionan protección fuera del marco tradicional de los estados nacionales? ¿Cómo el bienestar social de los jóvenes y de las personas mayores, que viven en la sociedad de origen, es garantizado cuando las personas que normalmente lo suministran han optado por emigrar? ¿Qué nuevos arreglos institucionales - o formas de protección social transnacional - están surgiendo como respuesta a estas dinámicas cambiantes?” (Levitt et al, 2016a: 3, traducción de los autores).

De acuerdo con Levitt (et al 2016a: 5-6), los estados, los mercados, las organizaciones de la sociedad civil, las redes sociales y las familias constituyen las fuentes de la protección social. Tales fuentes ofrecen (con diferentes grados de formalidad, estabilidad e incertidumbre) distintos tipos de protección social: protección para la vejez, la supervivencia y la incapacidad (pensiones, prestaciones monetarias, cuidado residencial, servicios funerarios, licencias por enfermedad, etc.); asistencia sanitaria; servicios y derechos para las familias (prestaciones familiares, licencia maternidad y paternidad, educación y cuidado niños, etc.); políticas activas del mercado de trabajo y asistencia para el desempleo; soporte para acceso a vivienda; y servicios educacionales (Levitt et al, 2016a 5-6, adaptado de OECD, 2007).

\subsection{Tipos de protección social transnacional: formal e informal}

La protección social puede ser considerada una forma de ensamblaje de elementos formales e informales profundamente vinculados entre si (Bilecen y Barglowski, 2015a). La protección social formal se vincula a las normativas estatales promovidas por fondos públicos, que son institucionalizadas a través de políticas públicas y legislaciones que operan en diversas escalas (nacionales, subnacionales y supranacionales) y aplicadas con múltiplos criterios de elegibilidad (Bilecen y Barglowski, 2015a: 207). La idea clásica de los derechos a la protección social formal se basa en la relación territorializada entre estados y ciudadanos o nacionales (De Guchteneire y Pécoud, 2009: 6). De este modo, existe un vínculo importante entre soberanía nacional y la protección social; en la medida en que se concibe que los recursos de bienestar son intercambiados por la lealtad interna de los ciudadanos y por medio de un cierre de las fronteras de los estados (Bommes y Geddes, 2000: 1). 
Esa narrativa sirve de justificación a la hora de limitar el acceso a la protección social a los no ciudadanos o no nacionales (los inmigrantes) y a los no residentes (los emigrantes). Además, esa visión clásica de la soberanía y de la protección social, dista mucho de la realidad de aquellas personas que transitan dentro de espacios transnacionales (Paul, 2017: 1-2).

Sin embargo, las relaciones entre estado y territorio han experimentado profundas transformaciones a lo largo de las últimas décadas, con importantes consecuencias para las nociones de soberanía, ciudadanía y pertenencia (Levitt y de la Dehesa, 2003: 588; Bauböck y Scholten, 2016). Con el objetivo de destacar el nexo del estado con las nuevas dinámicas de movilidad y diversidad de la población, emerge una pluralidad de adjetivos para la noción de ciudadanía (Faist, 2015: 27): posnacional (Soysal, 1994), múltiple (Held, 1995), multinivel (Yuval-Davis, 2000), flexible (Ong, 1999), transnacional (Bauböck, 2003), multicultural (Kymlicka, 1995) y anidada (Faist, 2001). No obstante, lejos de significar una garantía de vínculos más fuertes y estables entre ciudadanía, derechos y migraciones, esas nuevas formas de la ciudadanía se han visto atravesadas por un alto grado de diversidad y contingencia. Al mismo tiempo que se discute si la ciudadanía puede ser concebida más allá de los marcos del estado nacional, la disyunción entre la transnacionalización de la vida social y las formas de acceso a derechos formales de protección, comporta procesos de exclusión social. Así, por ejemplo, la simultaneidad (Levitt y Glick Schiller, 2004; Faist, 2000) que marca la experiencia de los transmigrantes puede llevar a una doble exclusión de los sistemas formales de protección social, en la medida en que muchos de ellos son ciudadanos no residentes en origen y residentes sin el estatus legal de ciudadanos en destino. En este escenario, los agentes gubernamentales tienden a tener pocos incentivos políticos para defender la garantía de derechos a los no ciudadanos, en la medida en que tal decisión puede generar reacciones negativas por parte de la opinión pública, tal y como se demuestra en los debates actuales en Estados Unidos y en la Unión Europea. De todos modos, el acceso (frecuentemente parcial) a la protección social formal a través de la residencia (denizenship) (Faist, 2015: 38; Hammar, 1990; Paul, 2017: 8) sigue siendo relevante y se asiste a una mayor tolerancia hacia la doble ciudadanía (Faist y Gerdes, 2008), tal y como se refleja en la proliferación de acuerdos multilaterales y bilaterales entre los estados nacionales (Avato et al, 2010). Estos acuerdos posibilitan la acumulación y portabilidad de derechos a lo largo del tiempo y a través de las fronteras (SabatesWheeler et al, 2011). La Unión Europea constituye un ejemplo paradigmático de institución transestatal, promotora de ese tipo de acuerdos. Otro ejemplo a destacar es el Acuerdo Multilateral Iberoamericano, que incluye 20 países latinoamericanos, España, Portugal y Andorra (Paul, 2017: 2).

Muchos estados han tomado medidas basadas en una concepción transnacional de la protección social dirigidas a su diáspora $\mathrm{y}$, en algunos casos, también a los miembros de las familias "left-behind". Lafleur y Lizin (2015) enumeran interesantes ejemplos, tales como el pionero caso de Mali y Francia, que desde 1979 cuenta con un acuerdo que facilita el pago al seguro social obligatorio en el país europeo y garantiza el acceso a la salud a familiares que viven en Mali. En la misma línea, los emigrantes mexicanos pueden pagar el Seguro Popular (seguro social obligatorio), tanto para poder beneficiarse ellos mismos cuando visiten el país, como para cubrir a los familiares que permanecen en México. La portabilidad de derechos también se refleja en otro tipo de medidas, como los convenios bilaterales o multilaterales de Seguridad Social (por ejemplo, el que firman España y Ecuador en 2007, o el Convenio Multilateral Iberoamericano de Seguridad Social, que ha sido firmado por 22 países y se encuentra vigente en 9 de ellos). Este tipo de 
convenios supone que las aportaciones realizadas a la seguridad social en un país puedan sumarse a las aportaciones efectuadas en otro país, de modo que la jubilación puede percibirse en cualquiera de los países involucrados (Redobrán y Paredes, 2017). A nivel binacional, también cabe destacar los acuerdos fronterizos, como por ejemplo los acuerdos bilaterales sobre atención sanitaria para las poblaciones migrantes ubicadas en la frontera argentino-boliviana (Courtis et al, 2010), o los programas y proyectos en materia social y cultural implantados en el espacio transfronterizo México-Estados Unidos (Gasca Zamora, 2002).

Además de esos acuerdos internacionales, muchos de los países emisores ofrecen, con formatos y grados de intensidad variables, políticas de protección y apoyo a los emigrantes (en las esferas de trabajo, salud y educación, pero también en el soporte al envío de remesas). Los casos de las políticas diaspóricas de Ecuador (Boccagni, 2011; Herrera y Grijalva, 2017), Filipinas (Alcid, 2003) y México (Waldinger, 2014) son algunos de los referentes en la literatura sobre el tema. No obstante, cabe destacar que una parte importante de esas dinámicas de cooperación interestatales y de acción transnacional de los estados están inseridas en un escenario marcado por dos lógicas potencialmente contradictorias: la de la securitización (preocupación de parte de los Estados receptores) y la del desarrollo (justificación para los Estados emisores) (Isotalo, 2009; Lazaridis, 2016).

Considerando el panorama delineado, es posible afirmar que la protección social formal se caracteriza por múltiples contingencias, incertidumbres y variables. Por consiguiente, muchos son sus vacíos y muy limitada es su capacidad a la hora de responder a las demandas específicas de la población migrante y de sus familias (Righard y Boccagni, 2015). De ese modo, la protección social informal se configura como un conjunto de recursos y estrategias "desde abajo", que cumple un rol crucial a pesar de ser menos visible que las dinámicas formales. La protección social informal emerge de las redes sociales y de los vínculos de cuidado fundamentados en lazos, frecuentemente asimétricos (por género y generación) de solidaridad, reciprocidad y obligaciones (Bilecen y Barglowski, 2015a: 207). En esas redes sociales y de cuidado tiene lugar el intercambio de apoyo emocional y el cuidado de niños, ancianos y enfermos, así como el envío y recepción de remesas monetarias (Boccagni, 2016: 3). De ese modo, la protección informal hace referencia, en gran medida, a las dinámicas de cuidado transnacional ya mencionadas en el apartado anterior de este artículo.

La PST informal es consistente con la idea de la migración como estrategia de sustento familiar; pero recalca, además, la interacción entre demandas, riesgos y provisiones territoriales y desterritorializadas (Boccagni, 2016). De ese modo, cabe destacar el rol fundamental que la protección social informal cumple en los casos de migrantes y no migrantes que viven en un contexto de ausencia (parcial o total) de recursos formales de protección (Bilecen y Barglowski, 2015a: 207). Boccagni llama la atención sobre la importancia de investigar cuán efectiva es la protección social informal en la reducción de las brechas existentes dentro de la protección social formal (Boccagni, 2016: 8). Bilecen y Barglowski (2015a: 204), por otra parte, argumentan que la protección social informal no es solo complementaria; sino que incluso puede resultar más eficaz a la hora de resolver determinadas demandas.

La protección social informal puede ser resultado de iniciativas de las asociaciones de inmigrantes que ofrecen servicios (funerarios, de salud y de educación) u organizan actividades culturales, religiosas y de recreación. Además, estas asociaciones también fomentan $\mathrm{y}$ canalizan tanto inversiones 
económicas como remesas sociales (ideas, prácticas y conocimientos que circulan como parte del conjunto de valores, estilos de vida, pautas de comportamiento y capital social) entre las comunidades de origen y de destino (Levitt, 1998, 2001; Lamba-Nieves, 2013; Levitt y Rajaram, 2013).

Los trabajos de Jones (2007) y Wright (2011) trascienden los efectos directos de las transferencias tangibles (principalmente remesas monetarias) y las remesas sociales (Levitt 1998, 2001) en la protección social, para poner de manifiesto cómo las migraciones internacionales impactan en la construcción y transformación de la noción de bienestar a través del espacio transnacional. Las informaciones que circulan son filtradas por los parientes y amigos que permanecen en las zonas de origen, dando lugar a narrativas sociales que sitúan el hecho migratorio como estrategia de mejora (o no) de las condiciones de vida. De ese modo, es posible tanto el enaltecimiento de la idea de sacrificio personal y sufrimiento por parte del migrante para lograr la mejora de la calidad de vida de los miembros del hogar, como destacar las consecuencias negativas que tiene la emigración para los hijos e hijas de padres ausentes y su correlación con la desintegración familiar.

Tras presentar las características principales de estos dos tipos de protección social, cabe resaltar que tal distinción solamente tiene fines analíticos (Boccagni, 2016: 2). La PST funciona como ensamblajes que atraviesan las fronteras nacionales, que combinan formatos de protección formal e informal y arreglos estatales y no estatales (Faist, 2013: 9). Por ejemplo, tal y como enfatiza la literatura sobre las cadenas globales de cuidado mencionada en el apartado anterior, contar con redes familiares que asuman el cuidado no remunerado es uno de los mecanismos esenciales que posibilita la emigración femenina. De ese modo, ese recurso familiar de carácter informal aumenta claramente las posibilidades del grupo familiar para poder acceder a recursos formales de protección social por medio de la movilidad internacional. Considerar la protección social como una combinación de elementos informales y formales es una de las características principales de la PST y permite llamar la atención sobre las interfaces entre las responsabilidades de los estados y de los individuos y sus familias. Además, subraya la tendencia actual de sobrecarga de los vínculos personales para la protección social, en pleno auge del neoliberalismo, de la austeridad en materia social y de la securitización de fronteras (Boccagni, 2011).

Con el fin de analizar las estrategias de prevención, gestión, reducción y superación de circunstancias adversas al bienestar generadas en las distintas esferas (Paul, 2017; Faist, 2013) por medio de la movilización de recursos formales e informales de PST, Levitt (et al 2016a) propone el modelo analítico del "entorno de recursos" (resource environment). Este consiste en: “(...) la combinación de todas las posibles protecciones disponibles desde las cuatro fuentes potenciales (Estados, mercados, tercer sector y redes sociales)" (Levitt et al, 2016a: 6)[1]. La disponibilidad de recursos de protección depende de la configuración de estas fuentes (capacidad de los Estados receptores y emisores, la presencia del tercer sector, la naturaleza del mercado), así como de las características de los individuos y sus familias (lugar de origen, lugar de residencia, género, raza, etnia, religión, ingresos, educación y la amplitud y profundidad de sus redes sociales) (Levitt et al, 2016a: 6-7). El modelo de "entorno de recursos" tiene una alta capacidad heurística y permite comprender cómo el acceso a la PST varía a través del espacio y el tiempo y a través de individuos y grupos.

La perspectiva de la PST se centra en la ausencia y 
debilidad de los sistemas de protección, que requieren que los migrantes (y sus familias) negocien y combinen la protección social informal (proporcionada por redes interpersonales) y formal (provista por el Estado y las organizaciones) (Levitt et al, 2016a: 3). Este enfoque tiene la capacidad de analizar las posibles limitaciones de los arreglos institucionales, así como de proponer políticas públicas capaces de promover entornos de recursos más eficientes (Levitt et al, 2016a: 3).

\section{Consideraciones Finales}

De acuerdo con Barglowski et al. (2015), la PST contempla el ensamblaje multisituado de elementos formales e informales que permiten el acceso a recursos para enfrentarse a los riesgos sociales en las distintas esferas (cuidado, trabajo, salud y educación) de lo que podríamos denominar derechos sociales. A partir del esfuerzo de investigadores como Peggy Levitt et al. (2016a) y Thomas Faist (2009; 2013), el enfoque de la PST propicia la relectura de los estudios anteriores, permitiendo tanto superar algunas de sus limitaciones (en particular la de los estudios circunscritos a la familia transnacional), así como impulsar el desarrollo de nuevas investigaciones, que puedan ser implementadas a partir de una mirada tanto ascendente (bottom-up) como descendente (top-down).

El estado de la cuestión que recoge este texto sitúa el potencial y la agenda de investigación propuesta por la PST. En una época marcada por el desajuste entre las escalas de la vida social y de la protección social, los estudios de la PST tienen la potencialidad de promover interfaces entre campos de estudios distantes hasta el momento, como el de las familias y cuidados transnacionales, el de los estudios de los regímenes de bienestar y el campo de las políticas sociales globales. De ese modo, se trata de una perspectiva aglutinadora, que ofrece herramientas para avanzar hacia una convergencia analítica. Así, la PST contribuye a mostrar los nexos entre movilidad, familia y bienestar social, problematizando críticamente las categorías y las lógicas discursivas que obstaculizan la superación de la inconsistencia entre vida social y protección social (Levitt et al, 2016a: 15-16). Por lo tanto, el programa de investigación de la PST hace hincapié en la necesidad de replantear los marcos analíticos a partir de los cuales aprehendemos los problemas sociales y sus posibles soluciones (Levitt y de la Dehesa, 2017: 1524).

Sin embargo, todavía quedan muchas preguntas en el aire. Ante la pertinente reflexión de Faist (2009) sobre de qué modo la cuestión social se reconfigura transnacionalmente, el autor afirma que aún en el supuesto optimista de que se asista en el futuro a la culminación de los derechos sociales transnacionales en términos de ciudadanía, no resulta concluyente que tales patrones estructurales globales llegaran a implicar procesos convergentes. Por consiguiente, concluye que la superación de las desigualdades sociales a nivel transnacional no puede basarse en la prospectiva de que los Estados nacionales traten de implementar los mismos derechos nacionales y sus respectivas regulaciones a escala transnacional. Faist (2009) se pregunta si es posible usar la noción de ciudadanía en un mundo sumamente fragmentado, sin caer en la frustración discursiva.

Una gobernanza global que a escala supranacional dirija el contenido de la agenda de una política social que tradicionalmente ha operado a escala nacional, plantea grandes retos. Supone articular las relaciones entre la globalización y los distintos modos de gobernanza, los regímenes de bienestar y las prácticas de redistribución y regulación de derechos. Más allá del reto de superar el "nacionalismo metodológico", partiendo de la base de que la protección social a menudo ha sido definida 
desde el Norte Global y desde principios europeos universalistas, la clave pasa por evitar aproximaciones exclusivamente top-down que reproduzcan paradigmas paternalistas claramente vinculados a nociones de la política social con un fuerte sesgo cultural (Deacon y Stubbs, 2013). Por ello, de acuerdo con Faist (2009), la inclusión de contribuciones de distintas regiones $\mathrm{y}$ contextos sociales en torno a las nociones de derechos sociales y la cuestión social transnacional resulta fundamental. De ahí que podamos concluir que el enfoque de la PST vinculado a los espacios sociales transnacionales que producen movimientos migratorios, e incorporando la agencia de individuos y grupos (en los términos que plantea el modelo analítico del "entorno de recursos"), ofrece sólidas premisas conceptuales, analíticas y metodológicas para lograr investigaciones que permitan superar tales principios universalistas y avanzar hacia la hibridez y la diversidad en cuanto a las estrategias, recursos e imaginarios que dan contenido a la protección social.

\section{Bibliografía}

Alcid, M. L. L. (2003): "Overseas Filipino workers: sacrificial lambs at the altar of deregulation", en E. Østergaard-Nielsen, ed., International migration and sending countries: Perceptions, policies and transnational relations, Basingstoke, Palgrave Macmillan, pp. 99 - 120.

Arriagada, I. (2010): "La crisis de cuidado en Chile", Revista de Ciencias Sociales, 27, pp. 5867.

Avato, J., J. Koettl, y R. Sabates-Wheeler (2010): "Social security regimes, global estimates, and good practices: The status of social protection for international migrants", World Development, 38, pp. 455-466.
Baldassar, L. y L. Merla, eds. (2014): "Families, Migration and the Circulation of Care: Understanding Mobility and Absence in Family Life", Nueva York y Abingdon, Routledge.

Baldassar, L., C. Baldock, y R. Wilding (2007): "Family Caring Across Borders. Migration, Ageing and Transnacional Caregiving", London, Palgrave McMillan.

Basch, L. G., N. Glick Schiller, C. SzantonBlanc. (1994): "Nations Unbound: Transnational Projects, Post-Colonial Predicaments, and Deterritorialized Nation-States", Langhorne, Gordon and Breach.

Bauböck, R. (1994): “Transnational Citizenship: Membership and Rights in International Migration", Aldershot, Edward Elgar.

Bauböck, R., y P. Scholten (2016): "Introduction to the special issue: «Solidarity in diverse societies: beyond neoliberal multiculturalism and welfare chauvinism»", Comparative Migration Studies, 4(1).

Bilecen, B., y K. Barglowski (2015): "On the assemblages of informal and formal transnational social protection", Population, Space and Place, 3(21), pp. 203-214.

Bilecen, B., G. Catir, y A. Orhon (2015): "Turkish-German transnational social space: stitching across borders", Population, Space and Place, 3(21), pp. 244-256. 
Boccagni, P. (2011): “Migrants' social protection as a transnational process: Public policies and emigrant initiative in the case of Ecuador", International Journal of Social Welfare, 20, pp. $318-325$

Boccagni, P. (2016): “Addressing transnational needs through migration? An inquiry into the reach and consequences of migrants' social protection across borders", Global Social Policy.

Bommes, M., y A. Geddes (2000): "Immigration and the welfare state", en Bommes, M. y A. Geddes, eds., Immigration and welfare: challenging the borders of the welfare state, London, Routledge, pp. 1-12.

Bonizzoni, P. (2015): "Here or There? Shifting Meanings and Practices in MotherChild Relationships across Time and Space", International Migration, 53(6), pp. 166-182.

Bryceson, D. F., U. Vuorela (2002): “The Transnational Family: New European Frontiers and Global Networks", Oxford y New York, Berg.

Courtis, C. et al. (2010): "Migración y salud en zonas fronterizas: el Estado Plurinacional de Bolivia y la Argentina", Santiago de Chile, CEPAL.

Daly, M. y J. Lewis (2000): “The concept of social care and the analysis of contemporary welfare states", The British Journal of Sociology, 51, pp.281-298.

De Guchteneire, P. y A. Pécoud (2009): “The UN convention on migrant workers' rights", en P.
De Gucheteniere, A. Pécoud y R. Cholewinski, eds., Migration and Human Rights. Cambridge: Cambridge University Press y UNESCO, pp. $1-44$.

Deacon, B., M. Hulse y P. Stubbs (1997): Global Social Policy: International Organisations and the Future of Welfare". London, Sage.

Deacon, B. y P. Stubbs (2013): Global social policy studies: Conceptual and analytical reflections", Global Social Policy, 13(1), pp. $5-23$.

Dobbs, E., y P. Levitt (2017): “The missing link? The role of sub-national governance in transnational social protections", Oxford Development Studies, 818 (January), 1-17.

Esping-Andersen, G. (1990): "The Three Worlds of Welfare Capitalism”, Cambridge, Polity Press.

Faist, T. (2000): “The Volume and Dynamics of International Migration and Transnational Social Spaces", Oxford, Oxford University Press.

Faist, T. (2001): "Social Citizenship in the European Union: Nested Membership", Journal of Common Market Studies, 39(1), pp. 39-60.

Faist, T. (2009): "The Transnational Social Question. Social Rights and Citizenship in a Global Context", International Sociology, 24(1), pp. 7-35.

Faist, T. (2013): “Transnational Social Protection: An Emerging Field of Study", Bielefeld, COMCAD Working Paper Series No. 113, Center on Migration, Citizenship and Development. 
Faist, T. (2014): "On the transnational social question: how social inequalities are reproduced in Europe", Journal of European Social Policy, 24(3), pp. 207-222

Faist, T. (2015): "Migración y teorías de la ciudadanía", en P. Mateos, ed., Ciudadanía múltiple y migración: perspectivas latinoamericanas, México D.F., Centro de Investigación y Docencia Económicas, Centro de Investigaciones y Estudios Superiores en Antropología Social, pp. 25-56.

Faist, T. y J. Gerdes (2008): "Dual citizenship in an age of mobility", en Bertelsmann Stiftung \& Migration Policy Institute, Delivering Citizenship, Gütersloh, Bertelsmann Stiftung.

Fraser,N.(2009): “Scales of Justice: Reimagining Political Space in a Globalizing World”, Nueva York, Columbia University Press.

Fuentes Gutiérrez, V. y B. Agrela Romero (2015): "Violencia institucional hacia las migrantes bolivianas. Marianismo y restricción a la movilidad", Athenea Digital, 15(4), pp. 81104.

GascaZamora,J.(2002): "Espaciostrasnacionales. Interacción, integración y fragmentación en la frontera México-Estados Unidos”, México D.F., Instituto de Investigaciones Económicas, Universidad Nacional Autónoma de México.

Gil Araújo, S. (2010): "Políticas migratorias, género y vida familiar. Un estudio exploratorio del caso español", en Grupo Interdisciplinario de Investigador@s Migrantes, coord., Familias, niños, niñas y jóvenes migrantes. Rompiendo estereotipos, Madrid, IEPALA editorial, pp. 8192.
Gil Araujo, S. y C. Pedone (2014): “Introducción. Familias migrantes y Estados: vínculos entre Europa y América Latina", Papeles del CEIC, 2014/2.

Glick Schiller, N., (2010): “A Global Perspective on Migration and Development Nina Glick Schiller", en Glick Schiller, N. y T. Faist, ed., Migration, Development, and Transnationalization: A Critical Stance, Nueva York, Berghahn Books, pp. 22-62.

Gonzálvez, H. (2016): "Los cuidados en la migración internacional", Sur, Revista Internacional de Derechos Humanos, 13(24), pp. 43-52.

Gregorio Gil, C. (1998): "Migración femenina. Su impacto en las relaciones de género", Madrid, Narcea.

Guaygua, G., ed., (2010): "La familia transnacional. Cambios en las relaciones sociales y familiares de migrantes de El Alto y La Paz a España”, La Paz, Defensor del Pueblo-PMHPIEB.

Hammar, T. (1990): "Democracy and the NationState: Aliens, Denizens, and Citizens in a World of International Migration", Aldershot, Gower.

Held, D. (1995): "Democracy and the Global Order: From the Modern State to Cosmopolitan Governance", Cambridge, Polity Press.

Hernández, I. y N. Baca (2016): “El trabajo de cuidado como elemento constitutivo de las comunidades transnacionales en la migración México-EU", Si Somos Americanos, Revista de Estudios Transfronterizos, 16(2), pp. 101-126. 
Herrera, G. (2004): "Elementos para una comprensión de las familias transnacionales desde la experiencia migratoria del Sur del Ecuador", en F. Hidalgo, ed., Migraciones. Un juego con cartas marcadas, Quito, ILDIS-Abya Yala, pp.215-232.

Herrera, G. (2011): "Cuidados globalizados y desigualdad social", Nueva Sociedad, 233, pp. 87-97.

Herrera, G. (2012): "Repensar el cuidado a través de la migración internacional: mercado laboral, Estado y familias transnacionales en Ecuador", Cuadernos de Relaciones Laborales, 30(1), pp. 139-159.

Herrera, V. R., y D. P. Grijalva (2017): "Políticas públicas para la población ecuatoriana migrante: ¿Hacia una protección social transnacional?”, GIGAPP Estudios Working Papers, 66-71, pp. 255-272.

Hochschild, A. R. (2000): “Las cadenas mundiales de afecto y asistencia y la plusvalía emocional", en H. Hutton y A. Giddens, eds., En el límite. La Vida en el Capitalismo Global, Barcelona, Tusquets Editores, pp. 187-208.

Hochschild, A.R. (2005): "Love and Gold", en Ricciutelli, L., A. Miles y M. H. McFadden, (eds.). Feminist Politics, Activism and Vision: Local and Global Challenges, Toronto, Zed/ Innana Books, pp. 34-46.

Hondagneu-Sotelo, P. (1994): “Gendered transitions: Mexican experiences of immigration", Berkeley y Londres, University of California Press.
Hondagneu-Sotelo, P. (1999): "Introduction. Gender and contemporary U.S Immigration", American Behavioral Scientist, 42, pp. 565-576.

Isotalo, R.(2009): "Politicizing the Transnational: On Implications for Migrants, Refugees, and Scholarship", Social Analysis, 53(3), pp.60-84.

Jones, R. C. (2014): "Migration and Family Happiness in Bolivia: Does Social Disintegration Negate Economic Well-being?", International Migration, 52, pp. 177-193.

Kilkey, M., y L. Merla (2014): "Situating transnational families' care-giving arrangements: The role of institutional contexts", Global Networks, 14(2), pp. 210-229.

Kofman, E. (2004) "Family-Related Migration: A Critical Review of European Studies". Journal of Ethnic and Migration Studies, 30(2), pp. 243262.

Kymlicka, W. (1995): "Multicultural Citizenship", Nueva York, Oxford University Press.

Lafleur, J. M. y O. Lizin (2015): “Transnational health insurance schemes: A new avenue for Congolese immigrants in Belgium to care for their realtives' health from abroad?", Transnational Studies Initiative Working Papers, Weatherhead Center for International Affairs Seminar at Harvard University. Disponible en web: http:// orbi.ulg.ac.be/bitstream/2268/172046/1/ Lafleur2015 tsiworkingpaper.pdf

Lagomarsino, F. y S. Castellani (2016): "The unseen protagonists. Ecuadorians' daughter between Ecuador and Southern Europe", Social 
Identities, 22(3), pp. 291-306.

Lamba-Nieves, D. (2013): "Hometown associations", en Ness, I., ed., Encyclopedia of global human migration, Nueva York, Wiley.

Landolt, P. (2006): "Modernidad tardía y migración transnacional: reflexiones conceptuales desde el caso de El Salvador", en Ardila, G., ed., Colombia: Migraciones, transnacionalismo y desplazamiento, Bogotá, Universidad Nacional de Colombia.

Lazaridis, G., ed., (2016): Security, Insecurity and Migration in Europe, Londres, Routledge.

Levitt, P. (1998): "Social remittances: migration driven local-level forms of cultural diffusion", International Migration Review, 32(4), pp. 92648.

Levitt, P. (2001): “The transnational Villagers", Berkeley y Los Ángeles, University of California Press.

Levitt, P., y R. De La Dehesa (2003): "Transnational migration and the redefinition of the state: Variations and explanations", Ethnic and Racial Studies, 26(4), pp. 587-611.

Levitt, P. y N. Glick Schiller (2004): "Conceptualizing simultaneity: A transnational social field perspective on society", International Migration Review, 38(3), pp. 1002-1039.

Levitt, P., y N. Rajaram (2013): "Moving toward reform? Mobility, health, and development in the context of neoliberalism", Migration Studies, 1(3), pp. 338-362.
Levitt, P., J. Viterna, A. Mueller y C. Lloyd (2016a): "Transnational social protection: setting the agenda", Oxford Development Studies, 45, pp. 2-19.

Levitt, P., E. Dobbs, S. Parella, A. Petroff y A. Viladrich (2016b): "The Role of Sub-National Governance in Global Social Protection: A Comparative Case Study of Spain and USA in Health Care". Comunicación presentada en la sesión "Global Social Protection and Migration: Reproduction of Inequalities or Safety Net?", Viena, III ISA Fórum de Sociología, Julio 2016.

Levitt, P., y R. de la Dehesa (2017): "Rethinking "transnational migration and the re-definition of the state" or what to do about (semi-) permanent impermanence", Ethnic and Racial Studies, 40(9), pp. 1520-1526.

Merla, L. (2014): "La circulación de cuidados en las familias transnacional", Revista CIDOB d'Afers Internacionals, 106-107, pp. 85-104.

Merla, L., y L. Baldassar (2016): "Response: Special Review Symposium, Transnational Families, Migration and the Circulation of Care", Papers: Revista de Sociologia, 101(2), pp. 275284.

Molanio, A. et al. (2012): "Cadenas globales de cuidados: síntesis de resultados de nueve estudios en América Latina y España", Santo Domingo, ONU Mujeres.

OECD. Organization for Economic Cooperation and Development (2007): "The social expenditure database: An interpretive guide", SOCX 1980-2003 
Ong, A. (1999): "Flexible Citizenship: The Cultural Logic of Transnationality", Durham, Duke University Press

Oso, L. (1998): "La migración hacia España de mujeres jefas de hogar", Madrid, Instituto de la Mujer, Ministerio de Trabajo y Asuntos Sociales, Serie Estudios $n^{\circ} 52$.

Oso, L., S. Parella, eds., (2012): "Inmigración, género y mercado de trabajo", Monografía de la Revista Cuadernos de Relaciones Laborales, Madrid, Universidad Complutense, Escuela de Relaciones Laborales, 30(1).

Parella S. y L. Cavalcanti (2008): “Aplicación de los Campos Sociales Transnacionales en los Estudios Sobre Migraciones", en Solé, C., S. Parella y L. Cavalcanti, orgs., Nuevos Retos Del Transnacionalismo en el Estudio de las Migraciones, Madrid, Ministerio de Trabajo e Inmigración de España, Observatorio Permanente De La Inmigración.

Parella, S. (2012): "Familia transnacional y redefinición de los roles de género. El caso de la migración boliviana en España”, Papers, 97(3), pp. 661-684.

Parella, S. (2016): "Introduction: The portability of care in an increasingly mobile world: Chains, drains and circulation". Papers: Revista de Sociologia, 101(2), 253-257.

Parreñas, R. (2005): “Long distance intimacy: Gender and intergenerational relations in transnational famílies", Global Networks, Vol. 5(4), pp. 317-336.
Paul, R. (2017): "Welfare without borders: unpacking the bases of transnational social protection for international migrants social protection for international migrants", Oxford Development Studies, 818, pp. 1-14.

Pedone, C. (2003): "Las relaciones de género en los grupos domésticos ecuatorianos dentro del contexto migratorio internacional hacia España", Treballs de la Societat Catalana de Geografía, 56, pp. 79-106.

Pedone, C. (2008): “'Varones aventureros' vs. 'Madres que abandonan', reconstrucción de las relaciones familiares a partir de las migraciones ecuatorianas", REMHU. Revista Interdisciplinar da Movilidades Humano, 30, pp. 45-64.

Pedone, C. y S. Gil Araujo (2008): “Maternidades transnacionales entre América Latina y el Estado español. El impacto de las políticas migratorias en las estrategias de reagrupación familiar", en Solé, C., S. Parella y L. Cavalcanti, orgs., Nuevos retos de transnacionalismo en el estudio de las migraciones, Madrid, Ministerio de Trabajo e Inmigración de España, Observatorio Permanente de la Inmigración.

Pedone, C., B. Agrela Romero y S. Gil Araujo (2012): "Políticas públicas, migración y familia: una mirada desde el género", Papers, 97(3), pp. 541-568.

Pérez Orozco, A. (2010): “Cadenas globales de cuidados. ¿Qué derechos para un régimen global de cuidados justo", Santo Domingo, UNINSTRAW. 
Pérez, R. y A. Neira (2017): "Las abuelas de la migración. Cuidados, reciprocidad y relaciones de poder en la familia transnacional", MIGRACIONES, 41, pp. 55-77.

Redobrán, V. y D. Paredes (2017): “Políticas públicas para la población ecuatoriana migrant: ¿hacia una prortección social transnacional?", GIGAPP Estudios Working Papers, 68, pp. 255272.

Righard, E y P. Boccagni (2015): "Mapping the theoretical foundations of the social workmigration nexus", Journal of Immigrant \& Refugee Studies, 13(3), pp.229-244.

Rivas, A. M. y H. Gonzálvez, eds. (2009): "Familias transnacionales colombianas", Madrid, La Catarata.

Sabates-Wheeler, R., J. Koettl y J. Avato (2011): "Social security for migrants: A global overview of portability arrangements", en SabatesWheler, R. y R. Feldman, eds., Migration and Social Protection: Claiming Social Rights beyond Borders, Londres, Palgrave Macmillan, pp. 115-144.

Sabates-Wheeler, R., y R. Feldman, eds. (2011): "Migration and social protection: Claiming social rights beyond borders", Basingstoke, Palgrave Macmillan.

Sørensen, N.N. (2007): "La vida de la familia transnacional a través del Atlántico: La experiencia de la población colombiana y dominicana migrante en Europa", Puntos de Vista, 9, pp. 7-28.
Soysal, Y.N. (1994): “The Limits of Citizenship”, Chicago, University of Chicago Press.

Speroni, T. (2017): "It is not only about access: Transnational Bolivian families in Barcelona and their meanings of social protection", Revista Internacional de Estudios Migratorios, 7(3), Número Especial, pp. 74 -103.

Tapia, M. (2011): “Género y migración: Trayectorias Investigativas en Iberoamérica", Revista Encrucijada Americana, 4(2), pp. 115147.

Vivas-Romero, M. (2017): "Who Cares for Those Who Cared? An Intersectional Ethnography of Global Social Protection Arrangements", Tesis Doctoral, Liège Université.

Waldinger, R. (2014): “The politics of crossborder engagement: Mexican emigrants and the Mexican state", Theory and Society, 43(5), pp. 483-511.

Wright, K. (2011): "Constructing human wellbeing across spatial boundaries: negotiating meanings in transnational migration", Global Networks, 12(4), pp. 467-484.

Yeates, N. (2006): "GSP Forum: New (?) Directions in Global Social Policy", Global Social Policy, 6(1), pp.16-20.

Yeates, N., ed. (2014): "Understanding Global Social Policy", Bristol, Polity.

Yuval-Davis, N. (2000): "Multi-layered Citizenship and the Boundaries of the NationState", International Social Science Review, 1(1), pp. 112-127. 
Recibido: 20 de noviembre de 2017

Aceptado: 04 de enero de 2018

\section{NOTAS}

[1] Traducción de los autores 